\title{
Acoustic shock waves in the solar chromosphere from millimeter observations
}

\author{
Maria A. Loukitcheva ${ }^{1,2}$, Sami K. Solanki ${ }^{1}$ \\ and Stephen White ${ }^{3}$ \\ ${ }^{1}$ Max-Planck-Institut für Sonnensystemforschung, D-37191 Katlenburg-Lindau, Germany \\ ${ }^{2}$ Astronomical Institute, St. Petersburg University, 198504 St. Petersburg, Russia \\ email: marija@peterlink.ru \\ ${ }^{3}$ Astronomy Department, University of Maryland, College Park, MD 20742, USA
}

\begin{abstract}
We argue that millimeter continuum observations promise to be an important diagnostic of chromospheric dynamics and the appropriate wavelengths to look for dynamic signatures are in the range $0.8-5.0 \mathrm{~mm}$. We have analyzed the millimeter intensity spectrum expected from the dynamic model of the solar non-magnetic atmosphere of Carlsson \& Stein $(1992,1995$, 1997, 2002, hereafter CS) together with the interferometric observations of the quiet Sun obtained at a wavelength of $3.5 \mathrm{~mm}$ with the Berkeley-Illinois-Maryland Array. Model radio emission at millimeter wavelengths is found to be extremely sensitive to dynamic processes in the chromosphere, if these are spatially and temporally resolved. The estimated millimeter brightness temperatures are time-dependent, following changes in the atmospheric parameters, and result in clear signatures of waves with a period of $180 \mathrm{~s}$ seen in the radio intensity as a function of time. At the same time, the interferometric observations of the internetwork regions reveal significant oscillations with amplitudes of $50-150 \mathrm{~K}$ in the frequency range $1.5-8 \mathrm{mHz}$. We give an estimate of the influence of the limited available spatial resolution of observations on the comparison with the predictions of dynamic models. We are able to establish a correspondence between the CS model predictions and the observational data if we assume that the horizontal coherence length of the oscillations is on the order of 1 arcsec.
\end{abstract}

Keywords. Sun: chromosphere, Sun: oscillations, Sun: radio radiation, shock waves

\section{Introduction}

The dynamic state of the solar chromosphere is now well established through observations made at different wavelengths stretching from the extreme UV via the visible to the far IR (e.g. Carlsson, et al. 1997; Judge, et al. 1997; Rutten \& Uitenbroek,1991; Kopp, et al. 1992) while the role played by chromospheric dynamics in structuring of this atmospheric layer is nowadays a subject of intense debate. The most prominent observational signature of chromospheric dynamics, namely the formation of Ca II $\mathrm{K}$ and $\mathrm{H}$ grains, has been explained in radiation hydrodynamic simulations of Carlsson \& Stein $(1992,1995,1997,2002)$, by upward propagating acoustic waves and the interactions between the shocks they produce in the middle chromosphere. According to the dynamic model, the chromospheric gas is mostly in a cool state with a transient temperature increase. Loukitcheva et al. (2004) proposed a new diagnostic that is highly sensitive to dynamic effects (sampling both the hot and the cool gas in the chromosphere) and may be provided by observations at millimeter wavelengths with an acceptable spatial resolution. In this contribution we present unique observations of chromospheric oscillations at $3.5 \mathrm{~mm}$ with the Berkeley-Illinois-Maryland Array and compare them with the intensity variations expected from the model of Carlsson \& Stein for mm wavelengths. 


\section{Results}

\subsection{Analysis of the CS model millimeter spectrum}

The response of the submillimeter and millimeter radiation to a time-series generated by Carlsson \& Stein was computed for 24 selected wavelengths in the mm range under the assumption of thermal free-free radiation by Loukitcheva et al. (2004). Waves with a period of approximately $180 \mathrm{~s}$ are clearly seen in the resulting model brightness temperature as a function of time at all considered wavelengths, but striking is the difference from one period of time to another due to shock wave interactions. Though the dominant frequency of the oscillations changes slightly with wavelength, for all $\mathrm{mm}$ wavelengths it lies in the range of 3 minutes. Judging from the brightness histograms at different $\lambda$ and the dependencies of the RMS brightness temperature and the dominant peak power on the wavelength (Fig. 1) we can identify the range $0.8-5.0 \mathrm{~mm}$ with a peak at $2.2 \mathrm{~mm}$ as the appropriate range of $\mathrm{mm}$ wavelengths at which one can expect the clearest signatures of dynamic effects.
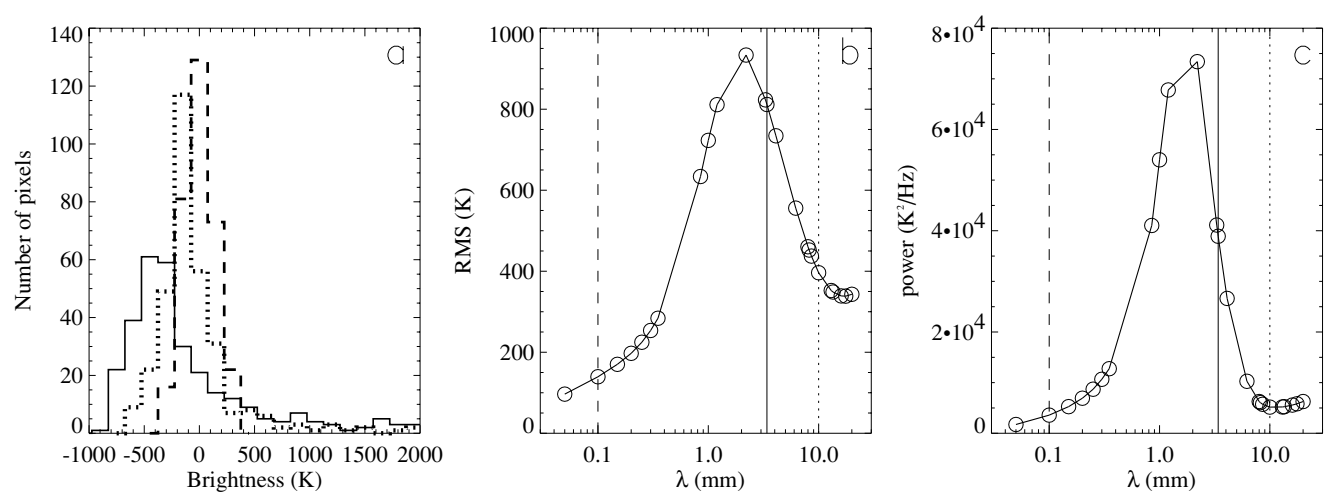

Figure 1. Characteristics of the millimeter spectrum expected from the CS model. a) Histograms of the model brightness for $\lambda=0.1 \mathrm{~mm}, 3.4 \mathrm{~mm}$ and $10 \mathrm{~mm}$, represented by dashed, solid and dotted lines, respectively. b) The RMS brightness temperature as a function of wavelength. c) The dominant peak power of the CS model spectral time series as a function of wavelength. Thin vertical lines mark the location of the 3 analysed wavelengths.

\subsection{Analysis of the BIMA observations at $3.5 \mathrm{~mm}$}

The only interferometer currently working in the $\mathrm{mm}$ range which is used for solar observations is the 10-element Berkeley-Illinois-Maryland Array (BIMA) operating at a wavelength of $3.5 \mathrm{~mm}$ (frequency of $85 \mathrm{GHz}$ ). With the BIMA data obtained on 31.08.2003 we have constructed two-dimensional maps of the solar chromosphere with a resolution of 10 arcsec, which represents the highest spatial resolution achieved so far at this wavelength for non-flare solar observations. The details of the restoration procedure and extensive tests of the sensitivity of the BIMA data to detection of dynamic signatures can be found in White et al. (2006).

Fourier and wavelet analyses of the BIMA data revealed the presence of intensity oscillations with RMS brightness temperature amplitudes of 50-150 K in the frequency range $1.5-8 \mathrm{mHz}$. We were able to distinguish between the properties of network and cell interiors and between bright and less bright parts of active regions and found that there is a tendency toward short period oscillations in the quiet Sun and longer periods 


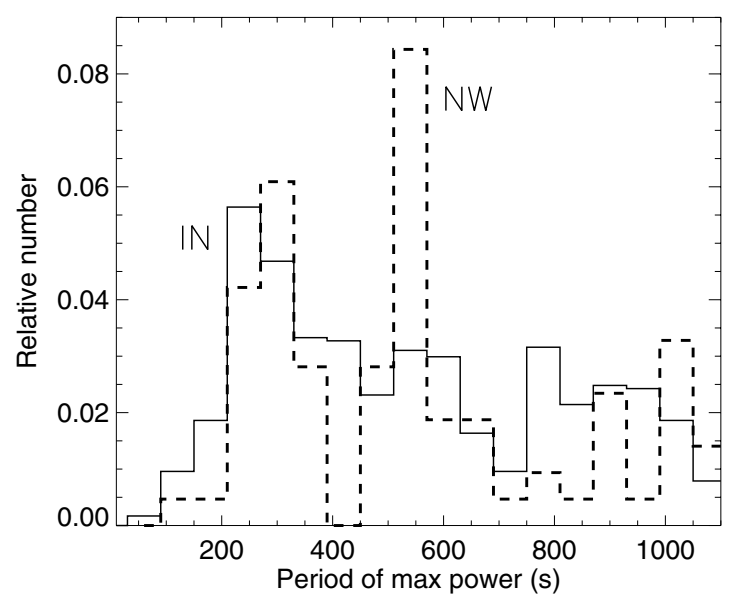

Figure 2. Histograms of the period of maximum power for the quiet Sun internetwork (solid line) and network (dashed line). For each pixel the most significant power is determined and at this period the pixel is included in the distribution.

in the active region, which is in good agreement with the results obtained at other wavelengths. The general difference in behavior between quiet and active regions is mirrored by internetwork and network regions in the quiet Sun, with the latter having a tendency to exhibit longer period oscillations (Fig. 2). Most of the oscillations are not steady harmonic waves but wave trains of finite duration lasting for typically 1-3 wave periods, as deduced from wavelet spectra.

\section{Discussion}

Due to the limited spatial resolution of 10 arcsec, a direct comparison of the observational data products (RMS values, histogram skewness, Fourier and wavelet spectra, etc.) with the corresponding products expected from the radiation hydrodynamic simulations of Carlsson \& Stein exhibits large differences. In particular, the RMS of the brightness temperature is nearly an order of magnitude larger in the model $(800 \mathrm{~K}$ at $3 \mathrm{~mm}$ ) than in the observations $(100 \mathrm{~K})$. Another difference is the absence of longer periods in the model power spectrum. But these discrepancies do not as yet rule out the CS models, since the direct comparison of the oscillations predicted by model and observed variations is inappropriate. The model is one dimensional and hence does not predict a coherence length of the oscillations. Searching for appropriate methods to compare the model and observations, we test the effect of limited resolution applied to the model predictions and to the observations.

We are able to obtain reasonable agreement between the observed millimeter oscillations and those predicted by the CS model if we model a resolution element by a statistical combination of 16 model sources of 3-min oscillations with random phase shift and 1 long-period source to account for the network emission. To evaluate the spatial scales of an oscillating source we carry out a complementary analysis and spatially smear the observational data further. We then use the resulting dependence of the RMS on the smearing to extrapolate to high spatial resolution in order to obtain a rough estimate 
of the expected RMS to be compared with the original simulations. The RMS of the individual frequencies was reconstructed from the wavelet power spectra.

According the obtained dependencies, a correspondence between the CS model predictions and the observational data can be established if we assume that the size of the coherence length of oscillating elements is of order of 1 arcsec. In this case the expected amplitude of oscillations is about $600 \mathrm{~K}$ and those estimated from the CS model millimeter spectrum have a similar value $(800 \mathrm{~K})$. However, our analysis has demonstrated that the currently available spatial resolution of 10 arcsec hinders a clean separation between cells and network and typically both network and internetwork areas contribute to the recorded radiation (see Loukitcheva et al. (2006)).

\section{Summary}

A direct comparison of the millimeter intensity spectrum expected from the dynamic model of Carlsson and Stein with the interferometric observations of the quiet Sun obtained at $3.5 \mathrm{~mm}$ with the Berkeley-Illinois-Maryland Array exhibits large differences. Nevertheless, we are able to establish a correspondence between the CS model predictions and the observational data under assumptions on the horizontal coherence length of the oscillations. In general, our results support the chromospheric model of CS, although there is a hint that the amplitude of the true oscillations is somewhat smaller than in the model. Our work shows the need for 2-D and 3-D models of chromospheric dynamics, which incorporate also a magnetic field.

Our work has demonstrated the potential of millimeter wavelength observations for studying of the solar chromosphere. Particularly exciting are the prospects of observing with the Combined Array for Research in Millimeter-wave Astronomy (CARMA) and the Atacama Large Millimeter Array (ALMA), which will provide a spatial resolution in the subarcsecond range. Both interferometers will be able to observe at a wavelength of $2.2 \mathrm{~mm}$ where the CS models predict that the largest observable effects will be seen.

\section{Acknowledgements}

The use of BIMA for scientific research carried out at the University of Maryland is supported by NSF grant AST-0028963. Solar research at the University of Maryland is supported by NSF grant ATM 99-90809 and NASA grants NAG 5-8192, NAG 5-10175, NAG 5-12860 and NAG 5-11872.

\section{References}

Carlsson, M., \& Stein, R.F. 1992, Astrophys. J., 397, L59

Carlsson, M., \& Stein, R.F. 1995, Astrophys. J., 440, L29

Carlsson, M., \& Stein, R.F. 1997, Astrophys. J., 481, 500

Carlsson, M., Judge, P. G., \& Wilhelm, K. 1997, Astrophys. J., 486, L63

Carlsson, M., \& Stein, R.F. 2002, Astrophys. J., 572, 626

Judge, P. G., Tarbell, T. D., \& Wilhelm, K. 2001, Astrophys. J., 554, 424

Kopp, G., Lindsey, C., Roellig, T.L., Werner, M.W., Becklin, E.E., Orrall, F.Q., \& Jefferies, J.T. 1992, Astrophys. J., 388, 203

Loukitcheva, M., Solanki, S.K., Carlsson, M., \& Stein, R.F. 2004, A\&A, 419, 747

Loukitcheva, M., Solanki, S.K., \& White, S. 2006, A\& $A$, submitted

Rutten, R.J., \& Uitenbroek, H. 1991, Solar Physics, 134, 15

White, S., Loukitcheva, M., \& Solanki, S.K. 2006, A\& A, submitted 